\title{
Incertidumbres en la estimación del periodo fundamental de terrenos inclinados
}

\author{
Uncertainties in the estimation of characteristic site period of sloping terrains
}

Fecha de entrega: 6 de diciembre 2016 Fecha de aceptación: 20 de abril 2017

\section{Edgar Giovanny Diaz-Segura}

Escuela de Ingeniería Civil, Pontificia Universidad Católica de Valparaíso, Av. Brasil № 2950, Valparaíso, Chile, edgar.diaz@pucv.cl

El periodo característico de un terreno $T_{0}$, es un parámetro ampliamente usado para definir el periodo de vibración en el cual una mayor amplificación sísmica puede ser esperada. Para terrenos inclinados, contrario a lo presentado en terrenos planos o de baja pendiente, la respuesta sísmica requiere de a lo menos un análisis bidimensional para determinar $T_{0}$. Si bien los antecedentes sobre comportamiento sísmico de terrenos inclinados representan una sólida base que permiten identificar algunas de las variables que condicionan la respuesta del suelo, actualmente no hay un criterio unificado respecto a la evaluación analítica o experimental del periodo característico de un terreno inclinado. En el presente estudio se realizó, usando el método de elementos finitos, un análisis paramétrico $2 \mathrm{D}$ de la respuesta sísmica de terrenos con inclinación superior a $15^{\circ}$, con el fin de identificar su periodo característico en campo libre. Asimismo, se realizaron mediciones usando la técnica de la relación espectral $H / V$, HVSR, en un terreno con ángulo de inclinación superior a $15^{\circ}$, cuyos resultados en términos del periodo fueron contrastados con la respuesta registrada en el análisis paramétrico realizado. A partir de los resultados obtenidos se observó que un punto localizado sobre un terreno inclinado puede registrar dos zonas de amplificación, cuyos máximos tienen asociado, independiente del ángulo de inclinación, igual periodo en cualquier punto a lo largo de la pendiente del terreno.

Palabras clave: periodo característico, terrenos inclinados, respuesta sísmica, método de relación espectral
The characteristic site period $T_{0}$, is a widely used parameter to define the period of vibration in which a greater seismic amplification can be expected. For sloping terrain, unlike what is seen with horizontal terrain, the seismic response requires at least a twodimensional analysis to determine $T_{0}$. Though the literature on the seismic behaviour of sloping terrain represents a solid basis for identifying some of the variables involved in the soil response, there are currently no unified criteria regarding an analytical or experimental evaluation of the characteristic site period for sloping terrain. The present study aims to use the finite elements method to conduct a $2 D$ parametric analysis of the seismic response of terrain with a slope of above $15^{\circ}$, in order to identify its characteristic freefield period. In addition, different measurements were taken using the HV spectral ratio method in a sloping terrain. These measurements were compared with the recorded response on the finite element parametric analysis. From the results, it is shown that any point on a sloping terrain features two amplification peaks, whose maximums are associated, independent of inclination angle of the terrain, with the same periods as any other point along the gradient of the terrain.

Keywords: characteristic site period, sloping terrains, seismic response, spectral ratio method

\section{Introducción}

El periodo característico de un terreno $T_{0}$, es un parámetro esencial para definir efectos de amplificación local producto de movimientos sísmicos del terreno, y proporciona una herramienta muy útil para definir el periodo de vibración en el cual una mayor amplificación de la señal puede ser esperada (Kramer, 1996). Diferentes códigos de diseño sísmico consideran de forma directa o indirecta $T_{0}$ como uno de los parámetros para definir los espectros de respuesta de diseño de acuerdo a la clasificación sísmica del terreno (BCJ, 1997; CEN, 2004; ICC, 2012).

Actualmente, la mayoría de los códigos de diseño sísmico no se pronuncian respecto a la validez o aplicación de sus criterios para el diseño de estructuras ubicadas en terrenos inclinados. El Código de Diseño Sísmico Francés (AFPS, 1995) y el Eurocódigo 8 (CEN, 2004), son quizás los únicos 
códigos que toman en consideración la inclinación del terreno, con el fin de incorporar en el diseño las potenciales amplificaciones producto de efectos topográficos. Aunque el código AFPS presenta consideraciones más precisas que el Eurocódigo 8, ambos incorporan un aggravation factor, $F$, definido en términos de la altura total del terreno y del ángulo de inclinación $\beta$. Sin embargo, el aggravation factor es independiente del período fundamental del suelo (CEN, 2004), por lo que es sólo usado como un factor de corrección escalar de las ordenadas del espectro de diseño elástico (Diaz-Segura, 2016).

Aunque existen algunos casos particulares como el de Tarzana Hill, en el cual se reportaron efectos topográficos importantes aún en pendiente baja (Bouchon y Barker, 1996), en terrenos con pendiente inferior a $15^{\circ}$, se ha observado que la influencia de la topografía es muy baja (AFPS, 1995; BRIJSCE, 1997; CEN, 2004; Assimaki et al., 2005). Por lo tanto, en terrenos con pendiente inferior a $15^{\circ}$ la determinación de su periodo característico, así como la definición de su clasificación sísmica podría realizarse de forma razonable aplicando criterios de clasificación y procedimientos de medición análogos a los usados en terreno planos.

A partir de las observaciones y análisis realizados por Idriss y Seed (1967) y Boore (1972), se han realizado diferentes estudios bidimensionales numéricos y analíticos con el fin de evaluar efectos topográficos (Çelibe, 1987; Buech et al., 2010; Assimaki y Jeong, 2013). Sin embargo, pocos estudios se han enfocado en evaluar de forma directa el periodo característico de un terreno con pendiente. Como un caso particular, Dakoulas y Gazetas (1985), mediante una aproximación bidimensional basada en el análisis conocido como shear beam, desarrollaron una solución analítica mediante la cual determinaron que $T_{0} \approx 2.61 \mathrm{H} / \mathrm{V}$, para el caso de una presa de tierra de sección triangular y altura $H$. De forma similar, basado en el estudio de Géli et al. (1988), Paolucci (2002) haciendo un análisis mediante el método Rayleigh, propone para el caso de un terreno con geometría triangular simétrica de altura $H$, que $T_{0} \approx$ $(H \tan \beta) /\left(0.7 V_{\mathrm{s}}\right)$. Por su parte, para el caso de un terreno inclinado, equivalente al caso de interés del presente estudio, Ashford et al. (1997), comparando la respuesta de un terreno inclinado en su punto más alto y la respuesta en campo libre tras el coronamiento, usan el término frecuencia topográfica, para definir la frecuencia de movimiento del terreno en la cual se produce la máxima amplificación topográfica. Esta frecuencia topográfica $f_{\mathrm{t}}$ tiene relación directa con los efectos de amplificación topográfica, sin embargo, difiere del concepto de frecuencia característica o fundamental del terreno. A partir de los casos analizados mediante modelación numérica, Ashford et al. (1997) definieron que $f_{\mathrm{t}} \approx V_{\mathrm{s}} / 5 H$.

Si bien los antecedentes sobre comportamiento sísmico de terrenos inclinados representan una sólida base que permiten identificar algunas de las variables que condicionan la respuesta del suelo, actualmente no hay un criterio unificado respecto a la evaluación analítica o experimental del periodo característico de un terreno inclinado. Existe una gran dificultad y un alto costo para la generación de modelos físicos, ya sea a pequeña o gran escala, que permitan obtener mediciones representativas de la respuesta sísmica de un terreno. Por lo tanto, con el fin de evaluar de forma individual y controlada las principales variables que condicionan la respuesta de un terreno, diferentes autores basan o complementan sus estudios en el análisis de propagación de ondas mediante modelación numérica (Wang, 2012; Foti et al., 2014; Matsushima et al., 2014; Diaz-Segura, 2016). De esta forma, la respuesta dinámica del terreno en campo libre puede evaluarse de forma paramétrica usando modelación numérica mediante la determinación de espectros de Fourier y/o espectros de respuesta en cualquier punto dentro del dominio de interés, lo cual a su vez permite identificar la influencia de cualquier variable en el periodo característico.

En el presente estudio, se realizó una evaluación del periodo fundamental de vibración del suelo para diferentes puntos localizados a lo largo de terrenos con ángulo de inclinación superior a $15^{\circ}$. Para ello se evaluó la respuesta dinámica del terreno mediante modelación numérica 2D en elementos finitos usando un suelo granular, apoyado sobre un basamento rocoso rígido sujeto a la propagación vertical de ondas de corte (in-plane $S V$-waves) (Ashford y Sitar, 1997; Bouckovalas y Papadimitriou, 2005; Tripe et al., 2013). Asimismo, tomando en consideración recomendaciones para registro de vibraciones en terrenos inclinados (Diaz-Segura, 2015), se realizaron diferentes mediciones en un terreno de alta pendiente usando de forma referencial el método de la relación espectral H/V 
(Nogoshi e Iragashi, 1978). Los resultados fueron a su vez contrastados con la respuesta registrada en modelos de elementos finitos bajo condiciones equivalentes.

\section{Características del modelo de elementos finitos usado}

Para evaluar la respuesta sísmica de un terreno inclinado de forma paramétrica, se realizó una modelación bidimensional mediante el método de elementos finitos usando una configuración topográfica como la mostrada en la Figura 1. Se construyeron modelos para valores de $H$ de 30, 60 y $90 \mathrm{~m}$, y ángulos de inclinación $\beta$ de $20^{\circ}, 30^{\circ}$ y $40^{\circ}$. La profundidad del basamento rocoso $H_{\mathrm{r}}$, se localizó a una profundidad fija de $30 \mathrm{~m}$.

El suelo fue modelado con comportamiento elástico lineal, lo cual adicionalmente permitió caracterizar el material principalmente con los parámetros de velocidad de propagación de ondas de corte $V_{\mathrm{s}}$ y razón de Poisson $v$. Se usaron suelos caracterizados con $V_{\text {s-medio }}$ de 150,350 y 500 $\mathrm{m} / \mathrm{s}, v$ de 0.3 y peso unitario $\gamma=19 \mathrm{kN} / \mathrm{m}^{3}$. El estado de esfuerzos iniciales se definió bajo una condición geostática usando un coeficiente de empuje lateral en reposo $k_{0}=0.4$ para todos los modelos.

A partir de un análisis de sensibilidad respecto a la influencia de la longitud de onda, para todos los modelos se seleccionó como señal sísmica un único pulso rectangular caracterizado con un periodo de $0.2 \mathrm{~s}$ y amplitud de $1 \mathrm{~g}$ (Diaz-Segura, 2016). La respuesta del terreno en campo libre, registrada durante $60 \mathrm{~s}$, se evaluó mediante de espectros de Fourier de aceleración determinados en 3 puntos localizados a lo largo de la pendiente del terreno, los cuales se muestran en la Figura 1, y que corresponde a un punto central $\mathrm{Y}$, y a dos puntos, $\mathrm{X}$ y Z, equidistantes del punto central, localizados sobre el talud a menor y mayor cota, respectivamente. Con el fin de determinar los periodos en los cuales se produce una mayor amplificación de la respuesta sísmica, se determinó la relación de amplificación, calculada como la relación entre los espectros obtenidos en campo libre y el espectro registrado en nivel del basamento rocoso (Kramer, 1996).

Para el modelo de elementos finitos fue usada una malla compuesta por elementos triangulares de 6 nodos garantizando un tamaño máximo de elemento igual a $\lambda / 15$, siendo $\lambda$ la longitud de onda asociada a una frecuencia máxima de $20 \mathrm{~Hz}$. Para reducir los efectos de reflexión de ondas en el área de interés, las fronteras laterales, de tipo viscoso, se localizaron a una distancia mínima, $I$ en la Figura 1, de ocho veces la longitud de onda máxima (DiazSegura, 2016).

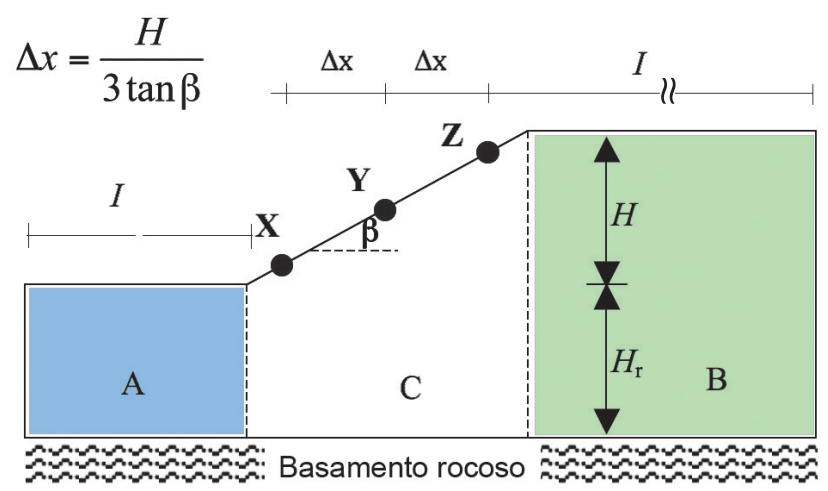

Figura 1: Características generales del caso tipo en estudio

\section{Identificación del periodo característico $T_{0}$ a lo largo de la pendiente de un terreno}

A partir de los resultados de los modelos realizados, se observó una respuesta dinámica característica en términos de la relación de amplificación, la cual se presenta para uno de los casos en la Figura 2, cuyos resultados muestran un comportamiento representativo de lo observado en los diferentes casos analizados. En la Figura 2 se muestra la presencia de dos zonas de amplificación registradas, las cuales se producen en periodos prácticamente iguales en cualquier punto a lo largo de la pendiente del terreno. Asimismo, se observó que para puntos cercanos al nivel más alto de la pendiente del terreno predomina la amplificación peak correspondiente al periodo de mayor magnitud, denominado $T_{0-B}$, lo cual se condice con las observaciones de Idriss y Seed (1967).

El comportamiento registrado, mostrado en la Figura 2, se puede explicar realizando un análisis simplificado centrando la atención en los periodos más que en la magnitud de la relación de amplificación. En un terreno como el mostrado en la Figura 1, el periodo $T_{0}$ del bloque B podría ser estimado de forma aproximada usando su correspondiente $V_{\mathrm{s}}$ y altura $H_{r}+H_{0}$ (Ashford et al., 1997),

$T_{0-\mathrm{B}}=\frac{4\left(H_{r}+H\right)}{V_{s}}$ 
(a)

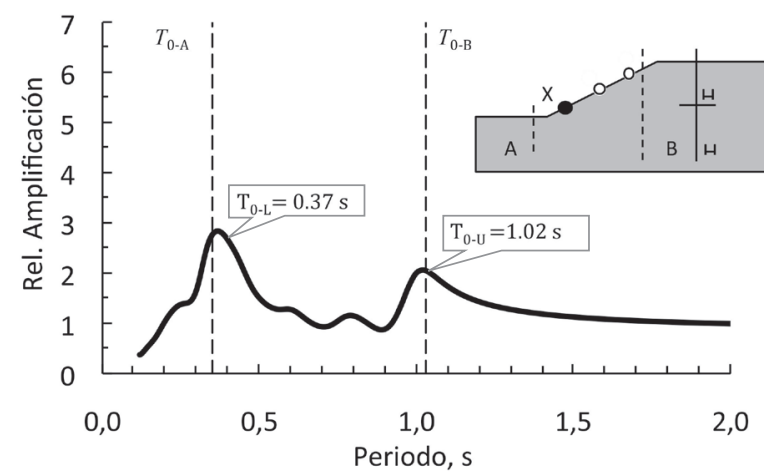

(b)

(c)
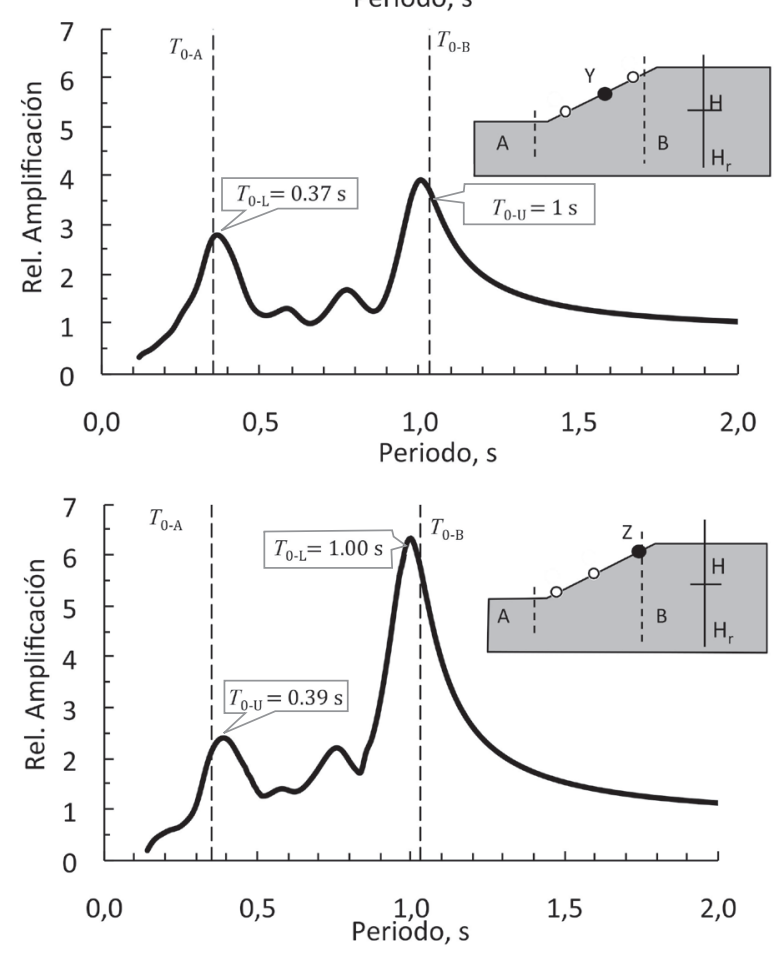

Figura 2: Relación de amplificación registrada a lo largo de la pendiente del terreno en a) X, b) Y y c) Z. Considerando $H_{\mathrm{r}}=30$ $\mathrm{m} ; H=60 \mathrm{~m}$; ángulo de inclinación $\beta=20^{\circ}, V_{s}=350 \mathrm{~m} / \mathrm{s}, T_{0-\mathrm{A}}^{\mathrm{r}}=$ $0.35 \mathrm{~s}$ y $T_{0-\mathrm{B}}=1.03 \mathrm{~s}$

Lo propio se podría extrapolar al bloque A usando la altura $H_{r}$,

$$
T_{0-\mathrm{A}}=\frac{4 H_{r}}{V_{s}}
$$

Dichos periodos se muestran con línea punteada en la Figura 2. Es claro que para el bloque $\mathrm{C}$ esta simplificación no sería válida, sin embargo, considerando la conexión inherente entre los tres bloques, los modos de vibración de cualquier punto en campo libre del bloque $\mathrm{C}$, estarían influenciados por el modo de vibración de los bloques A y B. Por lo tanto, la respuesta dinámica del bloque $\mathrm{C}$, estaría condicionado más que por el ángulo de inclinación del terreno, por la vibración de las masas de tierra localizadas a los extremos de la zona inclinada.
Lo anteriormente expuesto se observa para todos los casos analizados mediante la Figura 3, donde se muestra que los periodos que tienen asociado las mayores relaciones de amplificación registradas en los puntos $\mathrm{X}, \mathrm{Y}, \mathrm{y} \mathrm{Z}$, se ajustan muy bien con los periodos estimados usando las alturas de los bloques A y B (Figura 1), calculados de acuerdo a las expresiones (2) y (1), respectivamente. Considerando la clara relación registrada en los periodos, se tiene que la vibración del bloque $\mathrm{C}$, en términos de los periodos en los cuales se producen las mayores amplificaciones, está condicionada por los periodos de vibración del terreno circundante.

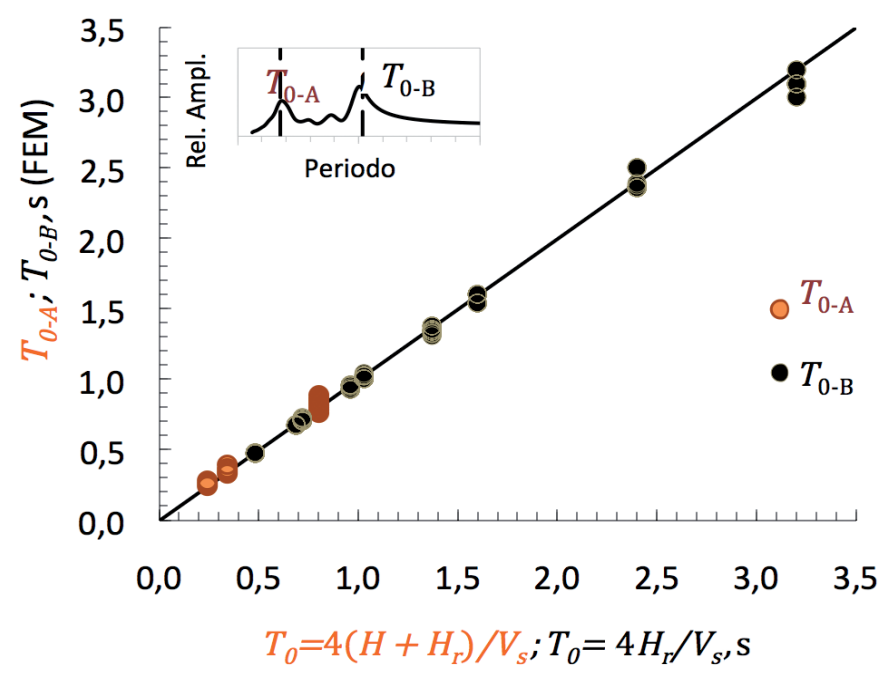

Figura 3: Periodo obtenido mediante modelo de elementos finitos versus $4 \mathrm{Z} / V_{\mathrm{s}}$ estimado para todos los casos analizados, $\mathrm{Z}$ $=H_{\mathrm{r}}$ y $Z=H_{\mathrm{r}}+H$

\section{Medición de la relación espectral H/V en un terreno inclinado}

Uno de los métodos más usados para medir $T_{0}$ es el propuesto Nogoshi e Igarashi (1971), que popularizó Nakamura (1989), quienes, a partir de medición de vibraciones ambientales en superficie, establecen que una pseudofunción de transferencia de las capas superficiales se puede definir mediante la relación entre las componentes horizontal y vertical de los espectros de amplitud de Fourier. El método denominado relación espectral H/V, HVSR, asume que las componentes vertical y horizontal del movimiento son radiales a nivel del lecho rocoso, es decir son iguales, y que sólo la componente horizontal de las ondas registradas en superficie es amplificada, por lo tanto, el periodo al cual se genera dicha amplificación es el periodo $T_{0}$. 
Si bien existen diferentes cuestionamientos al método HVSR respecto a la representatividad de la magnitud de la relación $\mathrm{H} / \mathrm{V}$ para evaluar la respuesta del terreno (Bard, 1998), la mayoría de los autores coinciden que el método es adecuado para registrar el periodo $T_{0}$, incluso en terrenos de alta pendiente para los cuales no habría impedimento para su uso (Lermo y Chávez-García, 1993; Chávez-García et al., 1996; Wood, 2003). Al margen de los cuestionamientos, en el presente estudio se usó el método de forma referencial con el fin de evaluar la forma de los registros de medición en diferentes puntos a lo largo de la pendiente de un terreno, con el fin de contrastar las mediciones con los resultados obtenidos mediante los modelos de elementos finitos.

Con el fin de evaluar la respuesta bajo condiciones reales, se realizaron diferentes juegos de mediciones, en un sector dunar de la ciudad de Concón. El terreno en dicho sector presenta una inclinación media de $28^{\circ}$, sin irregularidades importantes, y está compuesto por un depósito de arena de cementación media (Diaz-Segura, 2015). Las mediciones se realizaron en tres puntos a lo largo de la pendiente del terreno usando un sismógrafo de alta sensibilidad TrominoEngy, compuesto de tres sensores electrodinámicos ortogonales. Para cada punto de medición se usó un tiempo de registro de 30 minutos.

Basados en mediciones y en modelaciones, Matsushima et al. (2014) establecieron que, si bien el método de la relación espectral $\mathrm{H} / \mathrm{V}$ se puede emplear en zonas con heterogeneidades laterales, se debe tener presente que el registro de medición y su posterior análisis depende de la dirección de medición. Considerando lo anterior, se realizaron las mediciones en un terreno inclinado ubicando el equipo de manera que se registraran las vibraciones horizontales en dirección paralela y perpendicular a las curvas de nivel. Sin embargo, adicionalmente como se muestra en la Figura 4, se registraron vibraciones con un ángulo de $30^{\circ}$ y $60^{\circ}$ respecto de las curvas de nivel del terreno. En la Figura 4 se observa que independiente de la dirección de medición, es posible identificar los periodos que tienen asociado mayor relación HVSR. Las variaciones observadas entre las diferentes mediciones, puede atribuirse, además de a la pendiente del terreno, a la geometría que puede tener el basamento rocoso en la zona de medición (Matsushima et al., 2014). Por lo que siguiendo la recomendación de Matsushima et al. (2014), el análisis final de los resultados se realizó usando el registro de medición horizontal perpendicular a las curvas de nivel del terreno, es decir, dirección $0^{\circ}$.

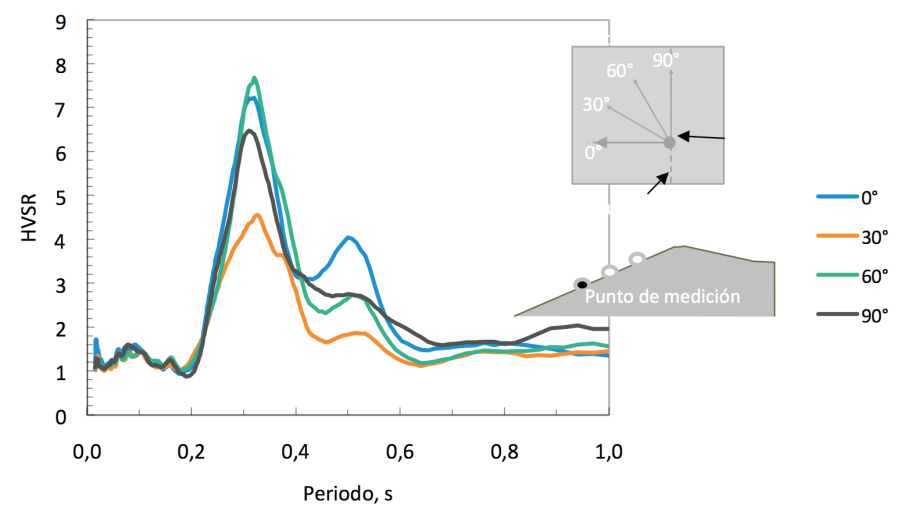

Figura 4: Influencia de la dirección de registro de vibración en la relación espectral HVSR medida

(a)

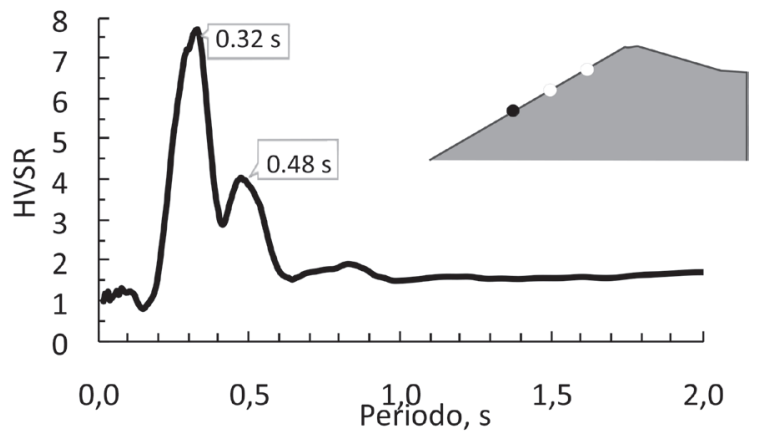

(b)

(c)
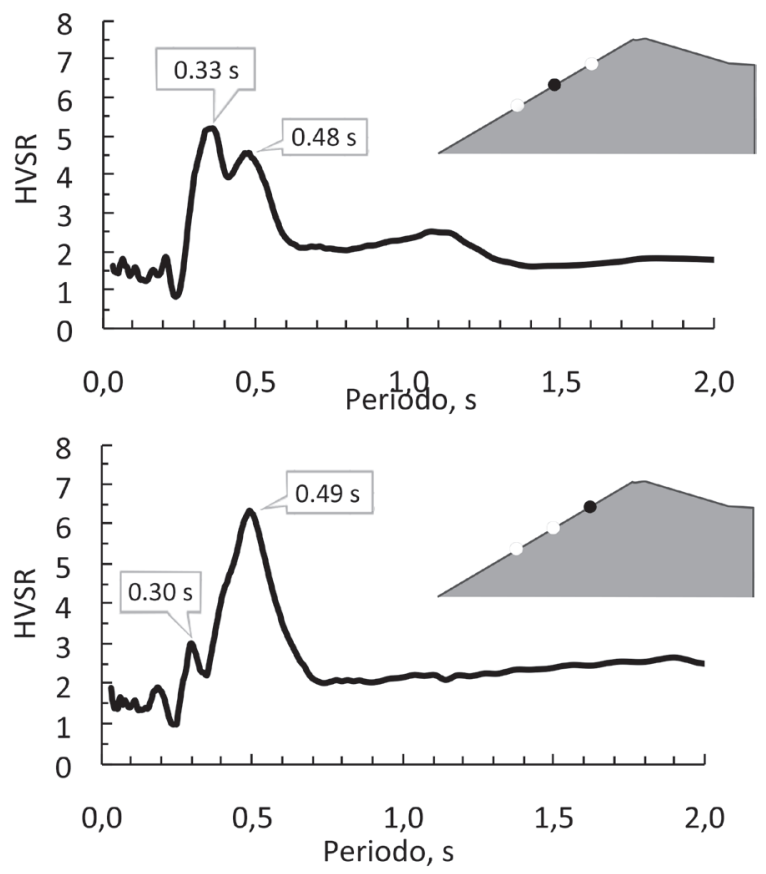

Figura 5: Medición de la relación HV para diferentes puntos a lo largo de la pendiente del terreno, a) X, b) Y y c) Z 
Las curvas de relación espectral registradas en los tres puntos de medición se muestran en la Figura 5, donde al igual que en el caso de la modelación, se registran dos amplificaciones cuyos periodos asociados se mantienen para los diferentes puntos de medición. Asimismo, se observa que, para los puntos localizados a mayor cota, predomina la relación espectral asociada al periodo de mayor magnitud. Lo anterior es equivalente a lo registrado mediante la modelación numérica, y da cuenta de que el periodo fundamental de vibración de un terreno inclinado no es único y está influenciado por la localización del punto de análisis y de los modos de vibración de los terrenos que circundan la pendiente del terreno.

\section{Conclusiones}

A partir de modelación mediante el método de elementos finitos $2 \mathrm{D}$ en terrenos con las diferentes configuraciones geométricas analizadas, al igual que mediciones de campo usando de forma referencial el método HVSR, se determinó que cualquier punto sobre un terreno inclinado registra dos amplificaciones principales, cuyos máximos tienen asociados los mismos periodos que cualquier otro punto localizado a lo largo de la pendiente del terreno. Por lo tanto, se podría preliminarmente indicar que, en un terreno inclinado con las características topográficas analizadas, sería más adecuado hablar de la presencia de dos periodos característicos, ya que las amplificaciones registradas, asociadas a dichos periodos, se mantienen a lo largo del terreno, alternándose los valores máximos de amplificación según su ubicación.

La vibración del terreno en la zona de pendiente está condicionada por la vibración del terreno circundante, por lo que los dos periodos registrados a lo largo del terreno, en donde se producen las mayores amplificaciones, son prácticamente iguales a los $T_{0}$ de las columnas de suelo a cada lado del terreno inclinado, calculados de acuerdo a la ecuación unidimensional $4 Z / V_{\mathrm{s}}$, siendo $Z$ la altura de la columna de suelo.

Si bien según los resultados del presente estudio en un terreno con la configuración mostrada en la Figura 1, se pueden presentar dos zonas de amplificación, desde un punto de vista práctico, es necesario evaluar si dicha condición produce que una estructura construida en un terreno inclinado tenga una demanda superior que pueda potencialmente condicionar su diseño.

\section{Agradecimientos}

El autor desea agradecer a la Pontificia Universidad Católica de Valparaíso, en el marco del Proyecto VRIEAPUCV No. 37/2016, por el apoyo financiero para el desarrollo del presente estudio. Asimismo el autor desea agradecer al Ingeniero Jorge Oviedo por el valioso apoyo brindado.

\section{Referencias}

AFPS (1995). Guidelines for seismic microzonation studies. AFPS/DRM, French Association for Earthquake Engineering

Ashford, S.A. and Sitar, N. (1997). Analysis of topographic amplification of inclined shear waves in a steep coastal bluff. Bulletin of the Seismological Society of America 87(3), 692-700

Ashford, S.A., Sitar, N., Lysmer, J. and Deng, N. (1997). Topographic effects on the seismic response of steep slopes. Bulletin of the Seismological Society of America 87(3), 701-709

Assimaki, D. and Jeong, S. (2013). Ground-motion observations at hotel Montana during the M 7.0 2010 Haiti earthquake: Topography or soil amplification?. Bulletin of the Seismological Society of America 103(5), 2577-2590

Assimaki, D., Kausel, E. and Gazetas, G. (2005). Soil-dependent topographic effects: a case study from the 1999 Athens earthquake. Earthquake Spectra 21(4): 929-966

Bard, P.Y. (1998). Microtremor measurements: a tool for site effect estimation? Second International Symposium on the Effects of Surface Geology on Seismic Motion, Yokohama, Japon, Vol. 3, 1251-1279

BCJ (1997). Structural provisions for building structures. Tokyo, Building Center of Japan

Boore, D.M. (1972). A note on the effect of simple topography on seismic SH waves. Bulletin of the Seismological Society of America 62(1): 275-284

Bouchon, M. and Barker, J.S. (1996). Seismic response of a hill: the example of Tarzana, California. Bulletin of the Seismological Society of America 86(1A): 66-72

Bouckovalas, G.D. and Papadimitriou, A.G. (2005). Numerical evaluation of slope topography effects on seismic ground motion. Soil Dynamics and Earthquake Engineering 25(7-10): 547-558 
Buech, F., Davies, T.R. and Pettinga, J.R. (2010). The little red hill seismic experimental study: topographic effects on ground motion at a bedrock-dominated mountain edifice. Bulletin of the Seismological Society of America 100(5A), 2219-2229

BRIJSCE (1997). Development of earthquake disaster prevention in urban area. Sectional committee report on risk evaluation of earthquake motion amplification. Building Research Institute and Japan Society of Construction Engineering (in Japanese)

Çelebi, M. (1987). Topographical and geological amplifications determined from strong-motion and aftershock records of the 3 March 1985 Chile earthquake. Bulletin of the Seismological Society of America 77(4), 1147-1167

CEN (2004). EuroCode 8: Design of structures for earthquake resistance - Part 1: General rules, seismic actions and rules for buildings. European Committee for Standardization, Brussels

Chávez-García, F.J., Sánchez, L.R. and Hatzfeld, D. (1996). Topographic site effects and HVSR. A comparison between observations and theory. Bulletin of the Seismological Society of America 86(5), 1559-1573

Dakoulas, P. and Gazetas, G. (1985). A class of inhomogeneous shear models for seismic response of dams and embankments. Soil Dynamics and Earthquake Engineering 4(4): 166-182

Diaz-Segura, E.G. (2015). Effect of MASW field configuration on the estimation of shear wave propagation velocity in a sloped terrain. Géotechnique Letters 5(1): 21-27

Diaz-Segura, E.G. (2016). Numerical estimation and horizontalto-vertical spectral ratio measurements of characteristic site period of sloping terrains. Géotechnique Letters 6(2): 176-181

Foti, S., Lai, C.G., Rix, G.J. and Strobbia, C. (2014). Surface wave methods for near-surface site characterization. CRC Press and Taylor \& Francis Group

Géli, L., Bard, P.Y. and Jullien, B. (1988). The effect of topography on earthquake ground motion: a review and new results. Bulletin of the Seismological Society of America 78(1): $42-63$
ICC (2012). IBC International Building Code. International Code Council, USA

Idriss, I.M. and Seed, H.B. (1967). Response of earth banks during earthquakes. Journal of the Soil Mechanics Foundation Division 93 (SM3), 61-82

Kramer, S.L. (1996). Geotechnical earthquake engineering. Prentice Hall, NJ

Lermo, J. and Chávez-García, F.J. (1993). Site effect evaluation using spectral ratios with only one station. Bulletin of the Seismological Society of America 83(5), 1574-1594

Matsushima, S., Hirokawa, T., De Martin, F., Kawase, H. and Sánchez-Sesma, F.J. (2014). The effect of lateral heterogeneity on horizontal-to-vertical spectral ratio of microtremors inferred from observation and synthetics. Bulletin of the Seismological Society of America 104(1), 381-393

Nakamura, Y. (1989). A method for dynamic characteristics estimation of subsurface using microtremor on the ground surface. Railway Technical Research Institute 30(1), 25-33

Nogoshi, M. and Igarashi, T. (1971). On the amplitude characteristics of microtremor (part 2). Journal of the Seismological Society of Japan 24(1), 26-40

Paolucci, R. (2002). Amplification of earthquake ground motion by steep topographic irregularities. Earthquake Engineering and Structural Dynamics 31(10): 1831-1853

Tripe, R., Kontoe, S. and Wong, T.K.C. (2013). Slope topography effects on ground motion in the presence of deep soil layers. Soil Dynamics and Earthquake Engineering 50: 72-84

Wang, L., Luo, Y. and Xu, Y. (2012). Numerical investigation of Rayleigh-wave propagation on topography surface. Journal of Applied Geophysics 86, No. 11, 88-97

Wood, C.M. (2013). Field investigation of topographic effects using mine seismicity. Doctoral thesis, University of Texas, Austin, USA 